\title{
RECOGNITION AND SELECTION OF SETTLEMENT SUBSTRATA DETERMINE POST-SETTLEMENT SURVIVAL IN CORALS
}

\author{
Lindsay Harrington, ${ }^{1,2}$ Katharina Fabricius,,${ }^{1,3}$ Glenn De’ Ath, ${ }^{1}$ And Andrew Negri ${ }^{1}$ \\ ${ }^{1}$ Australian Institute of Marine Science, Townsville, Queensland 4810, Australia \\ ${ }^{2}$ James Cook University, Townsville, Queensland 4811, Australia
}

\begin{abstract}
Habitat recognition and selective settlement by dispersive propagules greatly increases the post-settlement survival chances of sessile organisms. To better understand the key role some species can play in the structure of highly complex coral reef ecosystems, we compare the role of two independent, but sequential, processes: settlement choice and post-settlement survival. This study describes the chemical and physical recognition and ranking of specific settlement substrata by coral larvae. Several species of crustose coralline algae (CCA) are known to induce coral settlement; however they also employ physical and biological anti-settlement defense strategies that vary greatly in effectiveness. We examine the interactions between settling larvae of two common reef building coral species (Acropora tenuis and A. millepora) and five species of CCA (Neogoniolithon fosliei, Porolithon onkodes, Hydrolithon reinboldii, Titanoderma prototypum, and Lithoporella melobesioides) that co-occur on reef crests and slopes of the Great Barrier Reef, Australia. Distinct settlement patterns were observed when coral larvae were provided with a choice of settlement substrata. Settlement on the most preferred substratum, the CCA species T. prototypum, was 15 times higher than on $N$. fosliei, the least preferred substratum. The rates of postsettlement survival of the corals also varied between CCA species in response to their antisettlement strategies (shedding of surface cell layers, overgrowth, and potential chemical deterrents). Rates of larval settlement, post-settlement survival, and the sensitivity of larvae to chemical extracts of CCA were all positively correlated across the five species of CCA. Nonliving settlement substrata on coral reefs is sparse; consequently the fact that only a few CCA species (notably $T$. prototypum) facilitate coral recruitment, has important implications for structuring the reef ecosystem.
\end{abstract}

Key words: coral; coralline algae; defense; Great Barrier Reef, Australia; metamorphosis; recruitment; settlement; shedding; substrata; survival.

\section{INTRODUCTION}

The distribution and abundance of sessile organisms is often governed by the settlement of their mobile propagules. For marine organisms, larval settlement and early post-settlement survival can explain much of the variance in adult populations (Raimondi 1990, Palma et al. 1999). Settlement strategies of mobile larvae vary from passive, indiscriminate settlement to active substratum selection, which can help minimize the chances of settling in unsuitable habitats (e.g., Grosberg 1981). One of the recurrent themes of marine ecology has been the tendency to underestimate the capacity of larvae to influence their fate (Young and Chai 1985); however recent research suggests habitat selection by larvae at settlement has a strong influence on post-settlement survival and adult distribution patterns (Mundy and Babcock 1998, Baird et al. 2003).

Coral reefs are highly diverse marine communities constructed and dominated by sessile organisms that disperse via a planktonic larval stage in their early life

\footnotetext{
${ }^{3}$ Corresponding author. E-mail: k.fabricius@aims.gov.au
}

history. Microhabitats on coral reefs conductive to coral larval settlement and persistence are limited. Unoccupied primary substrata are rare and most other surfaces such as live corals, filamentous turf algae, and sediment are poor habitats for settlement and survival. Coral planula choose their site of permanent attachment based upon physical factors such as light, salinity, water motion, depth, surface orientation, and sedimentation (Maida et al. 1994, Mundy and Babcock 1998, Raimondi and Morse 2000). Living surfaces of nongeniculate or "crustose coralline" algae (CCA; Rhodophyta, Corallinaceae) can aid in the survival of settlers by excluding other space competitors and providing protection from sediment entrapped in turf algae (Babcock and Mundy 1996, Ruiz-Zarate et al. 2000). The suitability of a substratum as attachment site is however primarily determined by chemical and/or biological surface properties, such as surface films of algae, diatoms, or bacteria (Morse et al. 1988, Johnson et al. 1997, Raimondi and Morse 2000, Baird and Morse 2004).

Settlement and metamorphosis in many scleractinian corals is induced by external biochemicals (morphogens) associated with living CCA (Morse et al. 1988, 
Plate 1. Example of an anti-settlement defense mechanism in crustose coralline algae (CCA): epithallial shedding of $>80 \%$ of thallus surface, removing most of the two-day-old coral settlers on the CCA species Neogoniolithon fosliei in a single sloughing event. Two of the settlers are marked by arrows. Scale: $\sim 1: 1$. Photo credit: L. Harrington.

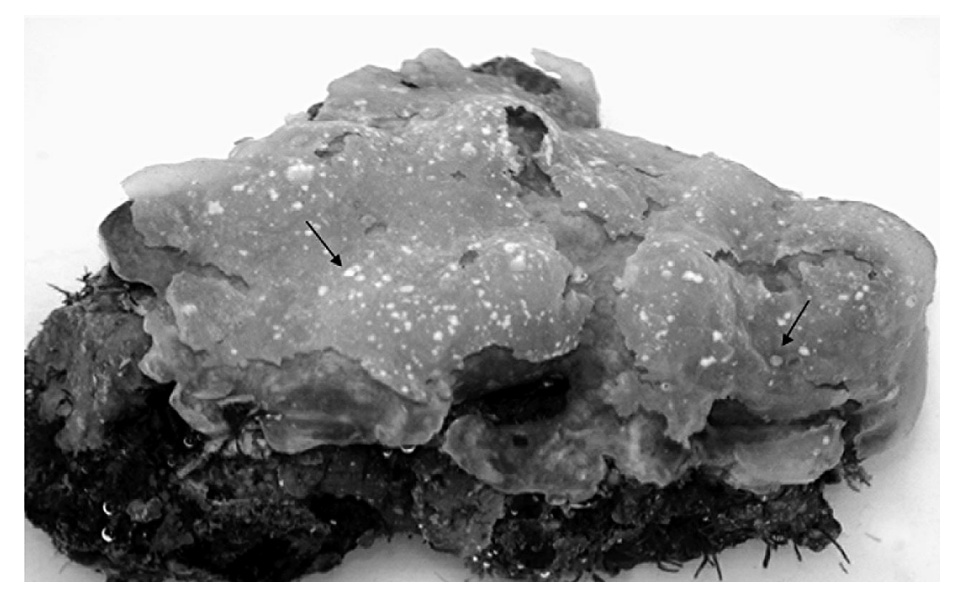

1996, Heyward and Negri 1999). These morphogens were identified as cell wall bound polysaccharides (Morse and Morse 1991). Although many species of CCA are capable of inducing metamorphosis in laboratory experiments, each species has specific characteristics that could influence its ecological relevance to habitat selection by the larvae. Larvae of the brooding Caribbean coral Agaricia humilis exhibited speciesspecific settlement preferences among five unidentified CCA species in laboratory experiments (Morse et al. 1988). Later research showed that larval settlement in this species was associated with the common CCA $\mathrm{Hy}$ drolithon boergensii (Raimondi and Morse 2000). A recent field study on the Australian Great Barrier Reef (GBR), has demonstrated a species-specific preference of broadcast-spawning corals to recruit on the coralline alga Titanoderma prototypum (R. Steneck, E. Turak, L. Harrington, and T. Done unpublished manuscript). The field observation of a high abundance of coral recruits associated with $T$. prototypum suggests this species may play a critical role in facilitating coral recruitment on the GBR.

Coral larvae may be induced to settle by properties of the substrata; however, subsequent mortality of recruits, caused by active substratum defenses, may result in the high rates of juvenile mortality. The potential for CCA to play a negative role in coral recruitment by increasing post-settlement mortality and reducing growth rates of coral recruits has not been considered. Many species of CCA eliminate newly settled organisms by shedding epithallial cells, i.e., sloughing (e.g., Keats et al. 1997). Overgrowth is also an important strategy of interference in the competition between space-limited organisms (Sebens 1986, Airoldi 2000). Thick CCA can easily overgrow newly settled corals (Maida et al. 1994, Dunstan and Johnson 1998), and even established adult corals (Antonius 2001). Chemical defense, in particular allelopathy, is a third widespread antifouling strategy used by many sessile organisms (Maida et al. 1995, Suzuki et al. 1998). Although chemical cues are known to induce settlement and metamorphosis of coral larvae on CCA, the potentially allelopathic nature of some of their metabolites have so far received little attention.

To better understand the key function some CCA species can play in the structure of coral reef ecosystems, we have examined the role and relationship of two independent but sequential processes: selective settlement and post-settlement survival of coral larvae. We quantified the settlement preferences of larvae of the two reef building corals Acropora millepora and A. tenuis on horizontal and vertical surfaces of living and dead CCA species, and on two inert substrata. We then quantified survivorship of newly settled corals on these substrata to determine if species-specific habitat selection influences post-settlement mortality levels. We also examined the chemically mediated induction of coral settlement by different species of CCA, and assessed the relationship of this process with that of selective settlement and post-settlement survival.

\section{Methods}

We examined how both physical and chemical attributes of CCA influence larval settlement and metamorphosis (Experiment 1a and b) and post-settlement survival of juvenile corals (Experiment 2) in a controlled laboratory environment. We also examined the presence of chemical inducers for metamorphosis (morphogens) in each species of CCA (Experiment 3 ). Experiments were conducted with two species of the coral genus Acropora, representing the dominant genus on the Great Barrier Reef (GBR) collected from three spawning events, and five species of common CCA found on offshore reefs of the GBR. Settlement experiments were performed in outdoor aquaria at the Australian Institute of Marine Science (AIMS, Townsville, Queensland), and at the Lizard Island Research Station (LIRS, Great Barrier Reef, Queensland).

\section{Obtaining coral larvae}

Experiments $1 a$ and 2.-Ten mature colonies of Acropora tenuis were collected prior to spawning from 
Nelly Bay, Magnetic Island $\left(19^{\circ} 10^{\prime} \mathrm{S}, 46^{\circ} 51^{\prime} \mathrm{E}\right)$. Spawning occurred on 2 November 2001.

Experiment 1b.-Six mature colonies of Acropora millepora were collected from Lizard Island $\left(14^{\circ} 40^{\prime} \mathrm{S}\right.$, $145^{\circ} 26^{\prime}$ E). Spawning occurred on 21 November 2002.

Experiment 3.-Six mature colonies of A. millepora (Ehrenberg 1834) were obtained from the Whitsunday Islands $\left(20^{\circ} 15^{\prime} \mathrm{S}, 148^{\circ} 50^{\prime} \mathrm{E}\right)$, which spawned at AIMS on 5 December 2001.

For all experiments the gametes were fertilized and larvae cultured as per Heyward and Negri (1999).

\section{Collection of non-geniculate crustose coralline algae}

Experiments 1a, 2, and 3.-CCA were collected from the slopes of Davies Reef ( $\left.18^{\circ} 50^{\prime} \mathrm{S}, 147^{\circ} 742^{\prime} \mathrm{E}\right)$ at 3-7 $\mathrm{m}$ depth. Five common species of CCA were collected on scuba using hammer and chisel: Hydrolithon reinboldii, Neogoniolithon fosliei (see Plate 1), Porolithon onkodes, Lithoporella melobesioides, and Titanoderma prototypum. The identity of each specimen was verified under a dissecting microscope using reproductive and vegetative morphological and anatomical features as diagnostic characters (Gordon et al. 1976, Adey et al. 1982). The epithallial surface area of each fragment was determined using the foil wrap technique (Marsh 1970; median area: $16 \pm 0.9 \mathrm{~cm}^{2}$, mean $\pm 1 \mathrm{SE})$. CCA fragments were maintained at AIMS in an outdoor 1000-L flow-through tank under a pivoted PVC trough that tipped $\sim 25 \mathrm{~L}$ of seawater every $\sim 2$ min to mimic a high-energy reef environment. One week after collection, the fragments were cleaned to remove all epibionts prior to experimental manipulation.

Experiment 1b.-The same CCA species (except for L. melobesioides) were collected at Lizard Island from the reef crest and slope between 3 and $7 \mathrm{~m}$ depths, and maintained in outdoor 25-L flow-through tanks at LIRS. Total thallus surface area averaged $11 \pm 0.4 \mathrm{~cm}^{2}$.

\section{Substratum-specific settlement rates}

Experiment 1a: Acropora tenuis larvae.-This experiment was performed to determine the effects of substratum type, the health of the CCA, and substratum orientation on settlement rates of A. tenuis larvae. Half of the CCA fragments of each species were kept alive, while the other half were killed by rinsing in fresh water for one hour and sun drying for five hours. Two additional treatments of inert substrata were prepared by cutting similarly sized fragments from unglazed fiveday conditioned terracotta and from dead skeleton of the massive scleractinian coral Porites sp. One-half of the live and dead fragments were embedded onto glass slides using underwater epoxy putty (AquaStik, Aquasonic, Ingelburn, New South Wales, Australia) allowing for vertical deployment. One replicate of each treatment (both live and dead vertical and horizontal fragments of five CCA species and two inert substrata) was placed in each of five aerated aquaria containing un- filtered seawater. These aquaria were placed within a large outdoor flow-through tank, acting as a water bath, under $90 \%$ shading to minimize overgrowth by filamentous algae. Nine days after fertilization, when the majority of larvae in the culture were competent to settle, $\sim 8000$ larvae were added to each of the five aquaria, where they were simultaneously presented with all substrata treatments. Three days after larval transfer, when many of the larvae had either settled or ceased swimming, the numbers of metamorphosed corals were determined on each fragment, using the criteria of Heyward and Negri (1999).

Experiment 1b: Acropora millepora larvae at LIRS.-To create stable horizontal settlement surfaces, all CCA fragments were embedded into plastic petri dishes with nontoxic underwater epoxy (Z-spar Splash Zone Compound, Kop-Coat, Pittsburgh, Pennsylvania, USA). Sixteen replicates of each of the CCA species, and fragments of terracotta tiles of similar sizes, were placed into eight replicate aquaria containing unfiltered seawater. At five days of age, 3000 larvae were added to each of the aquaria.

\section{Post-settlement survival}

Experiment 2: Survival of Acropora tenuis spat in the laboratory. - In order to assess early post-settlement survivorship, the number of A. tenuis spat needed to be increased on all substrata except for $T$. prototyp$u m$. To achieve this, all $T$. prototypum fragments were removed from the aquaria after three days of exposure to larvae. Within three more days, the number of settled and metamorphosed spat on all remaining substrata had increased by $19 \%$ (an additional 400 spat). After a total of six days exposure to larvae, all CCA fragments were transferred from the aquaria to perforated plastic trays and placed within the outdoor flow-through 1000-L tank with unfiltered seawater and the water-dumping PVC trough to remove sloughed cell layers. Survivorship of A. tenuis on the fragments was examined under a dissecting microscope daily for the first 30 days, and once more 6 months later.

\section{Investigating chemical responses}

Experiment 3: Larval metamorphosis in response to CCA morphogen concentrations.-We examined whether each CCA species contained extractable chemical inducers to trigger metamorphosis (morphogens) in the coral larvae tested. Sterile extracts from each of the CCA species, terracotta, and Porites sp. were prepared by individually grinding a known wet mass of chips of each substratum in methanol (HPLC grade). The slurry was passed through $0.45-\mu \mathrm{m}$, methanol washed nylon filters (47 mm diameter, Gelman Science, Ann Arbor, Michigan, USA) and the filtrate retained. The extraction process was repeated and the filtrates combined and evaporated to dryness under $\mathrm{N}_{2}$. The extracted residues were then resuspended in $0.2-\mu \mathrm{m}$ filtered seawater to a stock concentration equivalent to 
$500 \mathrm{mg} \mathrm{CCA} / \mathrm{mL}$ and stored frozen. Control extracts were prepared as described but with addition of either ground Porites or terracotta, or without the addition of ground substrata.

To test for settlement competency, larvae were exposed each day to $5 \times 5 \mathrm{~mm}$ chips of $P$. onkodes (Heyward and Negri 1999). High levels of metamorphosis $(>70 \%)$ were achieved after six days in 2001 and four days in 2002; hence the CCA extract induction experiments were performed with seven- and five-dayold larvae, respectively.

Larval metamorphosis assays were performed in sterile 10-mL wells (six-well culture plates, Nunc, Hongo, Bankyo-ku, Tokyo, Japan) maintained in a constant temperature room at $120 \mu \mathrm{mol}$ quanta $\cdot \mathrm{m}^{-2} \cdot \mathrm{s}^{-1}$ for $12 \mathrm{~h} / \mathrm{d}$, and strong fan-forced air flow to maximize gas exchange on the seawater surface in the wells. The temperature was set to $28-29^{\circ} \mathrm{C}$ in 2001 and $26-27^{\circ} \mathrm{C}$ in 2002. Coral larvae $(n=10-20)$ were introduced to each well containing $0.2-\mu \mathrm{m}$ filtered seawater and the CCA extract to a final volume of $10 \mathrm{~mL}$. The concentration range of CCA extract added to the wells was 0-50 $\mathrm{mg} \mathrm{CCA} / \mathrm{mL}$ seawater, with the concentration based upon the original wet mass of CCA extracted to enable comparison between species. For the highest extract concentration $(50 \mathrm{mg} / \mathrm{mL})$, the mass of organics added was $\sim 150 \mu \mathrm{g}$ total extract $/ \mathrm{mL}$. Six replicate wells were used for each treatment. Early-stage settlement and metamorphosis was assessed after $36 \mathrm{~h}$ using a dissecting microscope.

\section{Statistical analyses}

Experiment $1 a$ and $b$.-Log-linear models were used to analyze larval settlement rate as a function of settlement substratum (five CCA species and two controls), status of CCA fragment (live and dead), orientation (horizontal and vertical), and sampling year. The initial model involved main effects and all interactions. The final model was selected by backward elimination of nonsignificant terms $(P>0.05)$. Overdispersion was present in the data (McCullagh and Nelder 1989), and $F$ ratio tests based on the mean square deviance were used to assess the significance of effects.

Experiment 2.-For the survival data, we investigated how the instantaneous probability of larval survival at a given point in time (the hazard) varied with the treatments. The use of parametric and Cox proportional hazards models were assessed; however the model assumptions could not be satisfied for either approach. Since all censoring (discontinuation of observations on surviving larvae) occurred at the end of the experiment, it was possible to analyze the hazard for each period without the censoring resulting in biased estimates. The response variable was the number of deaths during a period divided by the number alive at the beginning of the period. Log-linear models were used with the hazard as the response, and the explanatory variables were settlement substratum, orientation, day, and aquaria nested in species. The variation due to aquaria was used as the error term for effects not involving day, and the mean deviance was used to assess all other effects (McCullagh and Nelder 1989). Partial effects plots were used to show the results; these plots show the effect(s) of one variable in a model adjusted for the effects of all others.

Experiment 3.-To investigate chemical induction of metamorphosis, the proportions of metamorphosed coral larvae were modeled using generalized additive models (Hastie and Tibshirani 1990) for the five species of CCA. Since the responses were proportions that varied smoothly with dose, we used binomial models with smoothing splines. The dose level at which the maximum response was observed was estimated from the fitted response curves. Confidence intervals for the maximum response dose for each species were obtained by taking bootstrap samples of the data $(N=1000)$, refitting the models, calculating the maximum response dose for each bootstrap sample, and taking the median as an estimate of maximum responses and 10th and 90th percentiles of the distributions as the end points of 90\% confidence intervals (Davison and Hinkley 1997). Differences between the species were assessed using permutation tests $(N=1000)$.

Synthesis.-The properties of the five species of CCA used in the three experiments were related to each other by examining correlations between the species effects across the three pairs of experiments.

\section{RESUlTS}

Experiment 1a and b: Substratum-specific settlement rates of Acropora tenuis and A. millepora larvae.Nine-day-old Acropora tenuis and five-day-old A. millepora larvae were observed to settle on all substrata tested, regardless of species, orientation, and health status, but the number of settling larvae varied greatly between the different treatments (Fig. 1). Log-linear analyses of the coral settlement rates onto CCA substrata revealed all interactions to be nonsignificant $(P>0.05)$. Differences in rates of settlement between the two sampling years were also nonsignificant, while all other main effects were significant: species $\left(F_{7,77}=\right.$ 7.12, $P<0.001)$, orientation $\left(F_{1,77}=13.5, P<0.001\right)$, and status $\left(F_{1,77}=41.0, P<0.001\right)$. Settlement varied greatly across the five species (Fig. 1), being 14.9 times higher $(95 \% \mathrm{CI}=(5.5,40.5))$ on $T$. prototypum (the best settlement inducer) than $N$. fosliei (the weakest settlement inducer). In all CCA species, living fragments induced greater settlement and metamorphosis than dead fragments, settlement being 9.4 (3.7, 23.6) times higher for live rather than dead substrata. On all substrata, settlement on horizontal surfaces was 2.8 $(1.5,5.1)$ times higher than on vertical surfaces.

Experiment 2: Survival of Acropora tenuis spat.Survival experiments revealed a very similar pattern to that observed for settlement. The variation in survival of coral spat between substrata was extreme (Fig. 


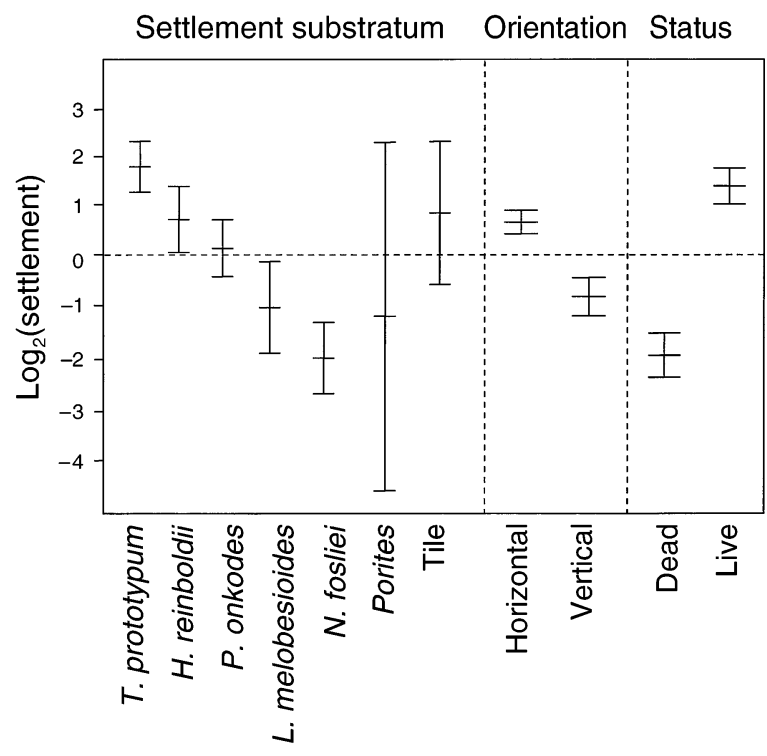

FIG. 1. Partial effects plots of settlement densities of coral larvae, showing the effects of various substrata (live and dead crustose coralline algae species (CCA) and inorganic substrata) at horizontal and vertical orientation (Experiment 1). The effects are plotted on $\log _{2}$ scale, and thus an increase or decrease by one unit corresponds to a doubling or halving in settlement numbers, respectively (e.g., settlement on live CCA is $2^{3.2}=9.2$ times as high as on dead CCA). Error bars represent \pm 1 SE.

2). Mortality was $100 \%$ on $N$. fosliei after three days, and $<50 \%$ on two other substrata ( $T$. prototypum and inert tile) after 22 days (Fig. 2). The log-linear analysis showed strong differences between substrata $\left(F_{7,584}=\right.$ 23.04, $P<0.001)$ and moderate differences due to days $\left(F_{(2,584)}=5.50, P=0.007\right)$, orientation $\left(F_{(1,584)}=5.48\right.$, $P=0.020)$, and the interaction between substrata and days $\left(F_{(13,571)}=4.47, P<0.001\right)$. This interaction was evident in the crossing of survival curves (Fig. 2), but

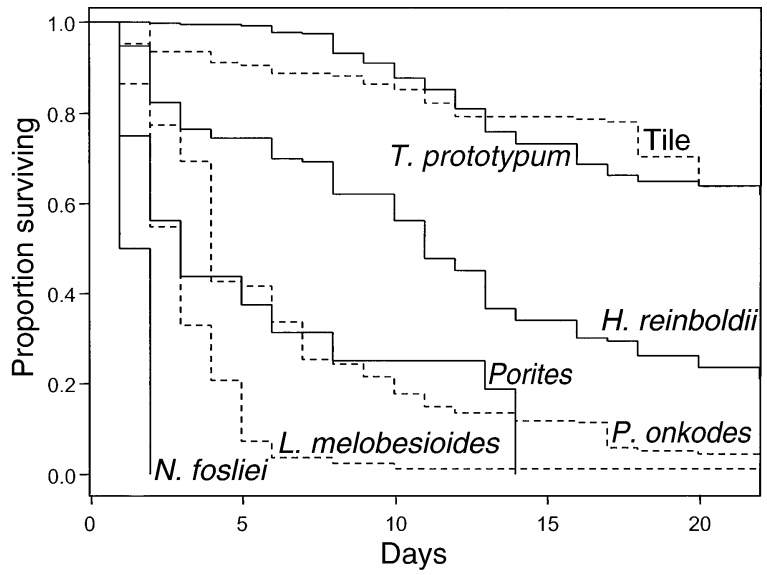

FIG. 2. Survival plots of coral settlers over 22 days (Experiment 2). The individual profiles correspond to proportions of surviving settlers on the five crustose coralline algae species and the two control substrata (Porites and tile) over time. was not particularly strong; thus averaged effects for substratum and days were obtained by dropping the interaction from the model. The probability of mortality (hazard) varied greatly across the five species, and was $36.5(20.1,66.1)$ times higher for $N$. fosliei than for $T$. prototypum: the latter did not differ from tile (Fig. 3). For orientation, vertical tiles exhibited higher relative hazards $(1.49,(1.13,1.97))$, and the relative hazard increased over the first 10 days but plateaued thereafter. After 240 days, survival of coral recruits on tiles and on $T$. prototypum was $20.1 \%(6.4,42.0)$ and $24.2 \%(8.2,52.1)$, whereas no survivors were observed on any of the other CCA species.

Three types of spat mortality were observed: (1) shedding of CCA thallus layers, thereby removal of the spat (see Plate 1), (2) overgrowth of the spat by CCA, and (3) mortality agents independent of visible/obvious CCA influences as estimated by mortality on the terracotta tiles (i.e., skeleton present, spat itself dead). Sloughing was seen in three CCA species: N. fosliei, $P$. onkodes, and $H$. reinboldii. In all colonies of $N$. fosliei, the sloughing of large sheets of epithallial cells removed $>50 \%$ of the thallus in single events (see Plate 1; also see Appendix: panel A). In contrast, small flakes or individual cells of thallus filaments were continuously shed in $P$. onkodes and $H$. reinboldii, resulting in continuous loss of spat over the course of the first five days (Fig. 2). After 22 days, the overall post-settlement mortality was greater in $P$. onkodes than in $H$. reinboldii, as some proportions of the surfaces appeared to remain unshed over extended periods of time

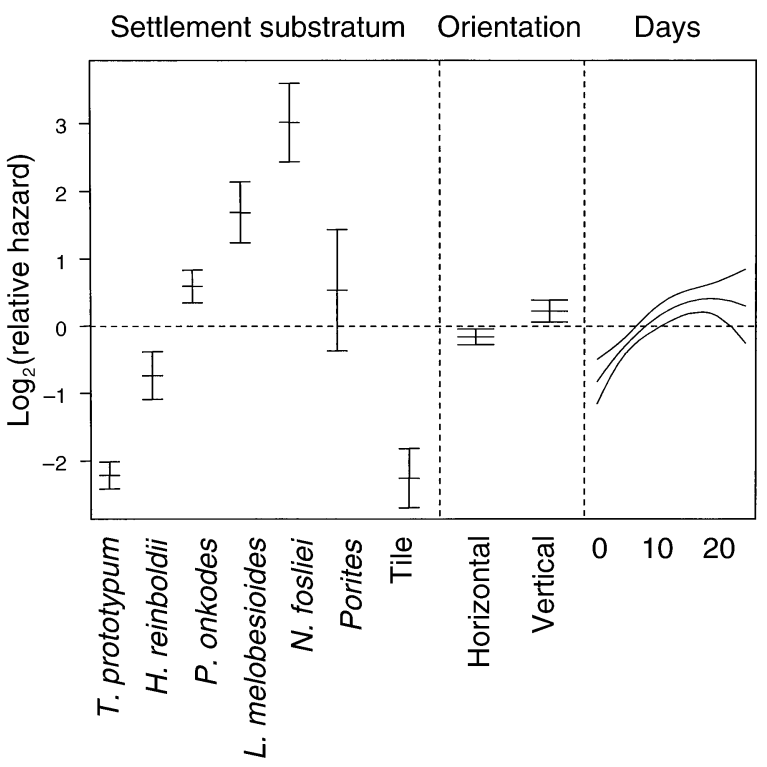

FIG. 3. Partial effects plots for relative hazard of coral spat (Experiment 2). The effects are for the substrata (crustose coralline algae species and inorganic substrata), orientation (horizontal and vertical), and the number of days since the start of the experiment. The effects are plotted on $\log _{2}$ scale; for interpretation see Fig. 1. Error bars represent \pm 1 SE. 

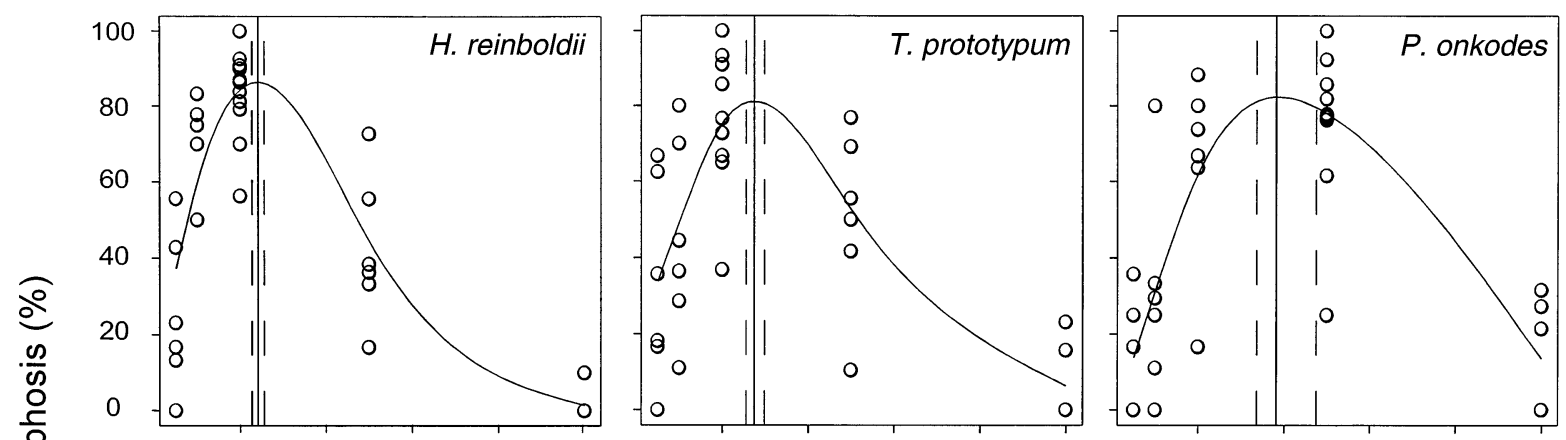

을
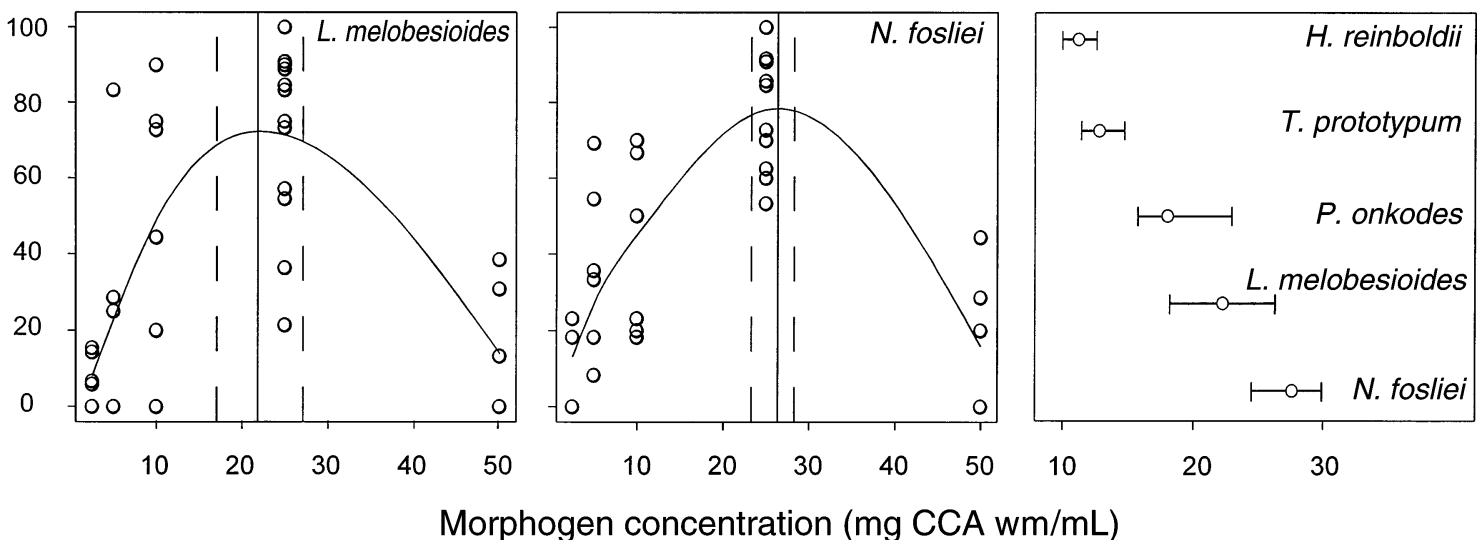

FIG. 4. Settlement and metamorphosis of coral larvae in response to concentrations of methanol-soluble extracts of crustose coralline algae (extract concentration based on $\mathrm{mg}$ CCA wet mass $[\mathrm{wm}] / \mathrm{mL}$ seawater; Experiment 3). Smoothing splines were fitted to the response data, solid vertical lines indicate curve maxima (determined by bootstrapping analyses), and dashed lines are 10\% and 90\% confidence percentiles. Nonoverlapping bars in the final summary plot indicate significant differences $(P<0.01)$ between species in the locations of the maxima.

in the latter species. Although L. melobesioides was not observed shedding, the initial drop in density during the first three days suggests that the spat may have been lost in an unrecognized sloughing event. T. prototypum was also not observed to shed.

Overgrowth was another observed cause of mortality in coral spat. $T$. prototypum and $P$. onkodes were able to completely overgrow spat aged 7-30 days (0.5-2 $\mathrm{mm}$ in diameter), employing two distinctly different strategies. In $P$. onkodes, the growing margin grew up and over the spat (Appendix, panel C). In T. prototyp$u m$, the growing margins approached from all directions in very thin layers, surrounding the spat and reaching around/above the basal plate of the coral until they had completely overgrown the entire coral (Appendix: panel B).

Experiment 3: Larval metamorphosis in response to CCA morphogen concentrations. - Extracts of each of the five species of CCA induced settlement and metamorphosis in A. millepora larvae, confirming that methanol-soluble morphogens were present in all CCA species tested. The maximum level of metamorphosis occurred at different concentrations for the five species (Fig. 4, permutation test, $P<0.001$ ), with levels of induction peaking at extract concentrations equivalent to $10-25 \mathrm{mg} \mathrm{CCA} / \mathrm{mL}$ (Fig. 4). The maxima for $H$. reinboldii and $T$. prototypum induction occurred at lower extract concentrations $(10 \mathrm{mg} \mathrm{CCA} / \mathrm{mL})$ than the remaining three species $(25 \mathrm{mg} \mathrm{CCA} / \mathrm{mL}$ ), whereas there were no differences within each of these two groups. At low to medium concentrations, larvae were actively swimming and appeared healthy, whereas at the highest concentration used (50 $\mathrm{mg} \mathrm{CCA} / \mathrm{mL})$, some of the larvae appeared unusually elongated and immobile. Control extracts (from terracotta, Porites, or no substratum) induced no metamorphosis over the concentration range examined.

\section{Synthesis of the three experiments}

Pairwise plots of the estimated effects for the five species showed strong positive relationships between CCA extract-induced metamorphosis, settlement, and survival (Fig. 5). High levels of coral settlement were related to high levels of survival (low relative hazards, $r=0.99, P<0.001)$ and to high sensitivity to the corresponding CCA chemical inducer $(r=0.91, P<$ $0.030)$. Survival was highest on CCA species that induced highest levels of metamorphosis at low concentration of CCA extracts $(r=0.92, P<0.026)$. The least chosen settlement substrata required higher concentrations of the species-specific CCA extract and exhibited low post-settlement survival, indicating that 

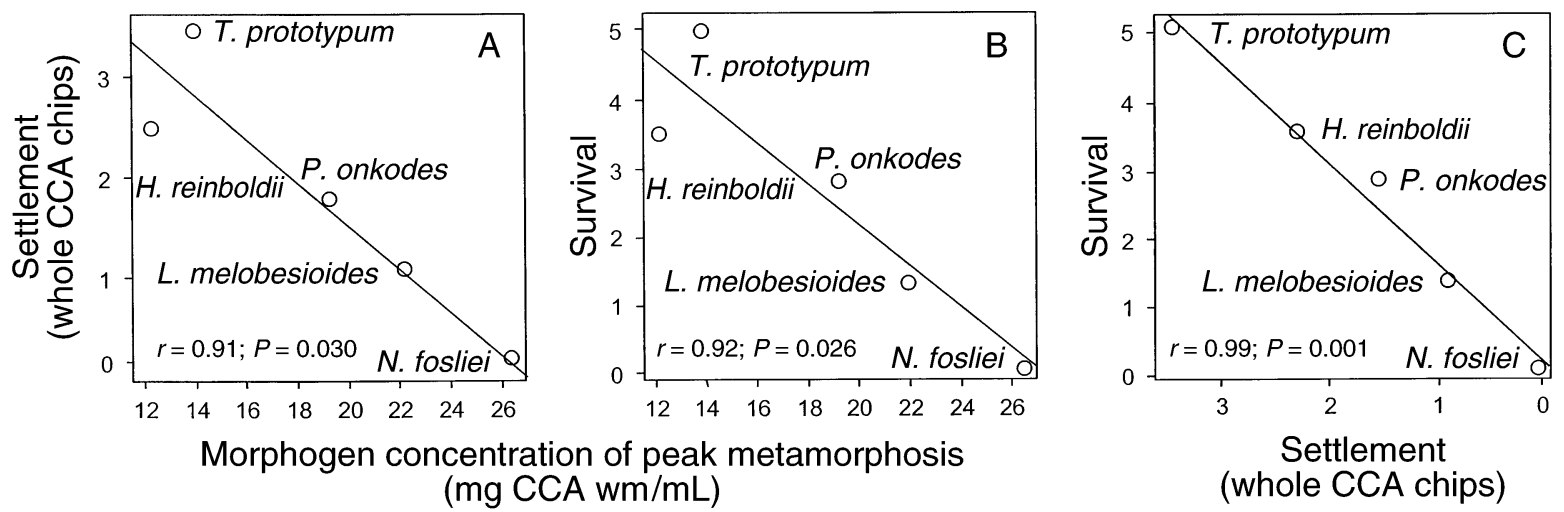

FIG. 5. Relationships between relative effects of sensitivity to chemical induction extract concentrations (Experiment 3), settlement (Experiment 1), and survival (Experiment 2) of coral larvae for the five species of crustose coralline algae (CCA). (A) Rate of settlement on whole live CCA fragments vs. sensitivity to CCA extract concentration. (B) Survival vs. sensitivity to CCA extract concentration. (C) Survival vs. settlement on whole, live CCA fragments. Sensitivity to chemical induction of metamorphosis was estimated as the concentration (\%) at which peak induction occurred (Fig. 4), settlement effects were estimated as the relative probability of settlement $\left(\log _{2}\right)$ (Fig. 1), and survival effects were estimated as the inverse of the relative hazard $\left(\log _{2}\right)$.

early presettlement inducers and behaviors allow larvae to select and settle on substrata, thus enhancing their future survival probabilities.

\section{DisCUSSION}

CCA have previously been identified as preferred recruitment substrata for scleractinian corals (Morse et al. 1988, Raimondi and Morse 2000). Our research confirms this role for certain key CCA species of the Great Barrier Reef but not for other, more abundant, CCA species. We demonstrated that coral larvae are able to recognize and choose CCA species that have the least effective antifouling defenses. Active selection of permanent attachment onto certain substrata leads to an increase in survival over the first four weeks following settlement. The highest rates of settlement on $T$. prototypum coincided with lowest post-settlement mortality, and settlement was triggered by one of the lowest CCA extract concentrations. In contrast settlement induction and survival was lowest on the most abundant CCA species in the GBR, N. fosliei and P. onkodes. Therefore, a few select species of CCA can greatly contribute to controlling the fine-scale distribution patterns of corals within a reef ecosystem.

\section{Settlement cues for coral larvae}

Settlement rates in A. tenuis and A. millepora clearly differed depending on: (1) CCA species, (2) orientation of the substrata, and (3) whether the CCA was alive or not. Strong chemical inducers for settlement and metamorphosis (morphogens), such as the cell wall-bound polysaccharide identified from $H$. borgesenii (Morse and Morse 1991) should facilitate the selection of settlement sites that enhance post-settlement survival. Previous studies investigating the source and potency of coral morphogens have been performed using substrata or CCA extracts in isolation (Morse et al. 1988,
1996, Morse and Morse 1991, Heyward and Negri 1999). Those experiments demonstrate that extracts of most species of CCA tested contain morphogen(s) able to induce high levels of settlement and metamorphosis of coral larvae within 24 hours. Our results also show that, each of the CCA species tested contained a strong methanol-soluble morphogen, and that the potency of the CCA extract differed between species. The comparison of extract potencies may not reflect field behavior due to: potential differences between species in the solubility of active compounds, their surface areamass ratios, and possible inhibition by co-extracted metabolites. When provided a choice of settlement substrata, larvae preferentially settled upon $T$. prototypum, the species that, along with $H$. reinboldii, exhibited the most potent CCA extract. Strong correlations between settlement preference on CCA fragments and extract potency were observed for all CCA species tested. These results suggest that coral larvae may be able to recognize subtle differences in chemical signatures or respond to different concentrations of morphogen on the surface of the CCA and use these signals when selecting their attachment site. Further research into the identity and specific concentrations of morphogen(s) associated with each of the CCA species is needed to confirm this.

Physical factors also contributed to determining the choice of attachment site. Settlement by A. tenuis larvae was significantly higher on horizontal opposed to vertical surfaces. This confirmed previous observations that larvae prefer upper surfaces for settlement, so long as these surfaces are free of sediment, algal growth, and grazing (Mundy and Babcock 1998). Physical conditions such as light and sedimentation are known to strongly influence settlement orientation in corals. For example, preferences shift from under surfaces in shallow water or at high light levels, to vertical surfaces 
and then upper surfaces in deep water (Babcock and Mundy 1996, Baird and Hughes 2000). Again, this settlement behavior has selective advantages, despite the slower growth rate in a shaded position, as it reduces post-settlement mortality by grazing, algal growth, and sedimentation (Raimondi and Morse 2000).

The clear preference for larvae to settle on live CCA rather than dead CCA of the same species, indicates that chemical or biological properties of living CCA surfaces are more effective in inducing coral settlement, or that compounds released upon death inhibit settlement to some degree. This is supported by previous laboratory assays where larvae were induced to metamorphose by dead CCA but attached to the polystyrene walls of the test wells, instead of the dead CCA chips (Heyward and Negri 1999). Clean terracotta tiles do not contain chemical morphogens and need to be "conditioned" in seawater to enable the establishment of a microbial/algal biofilm before settlement takes place. The terracotta tiles used in these experiments were conditioned in unfiltered seawater for five days, and presented only a very immature biofilm; nevertheless they were a highly preferred settlement substratum, possibly due to early biofilm development on the tile surface (Webster et al. 2004). Alternatively, the larvae may have encountered water-borne CCA morphogens, or morphogens on nearby CCA (possibly through an amplifier pathway similar to that described in the larvae of the abalone Halotis rufescens; Morse and Morse 1984), and then moved along to metamorphose on the inert surface of the tile. Living CCA surfaces can aid in the survival of settlers, by excluding other space competitors, and, by providing protection from turf algae and the sediment entrapped in turf algae (Babcock and Mundy 1996, Ruiz-Zarate et al. 2000). However, while CCA can reduce the mortality of newly settled corals, these recruits also need to survive the defense strategies of the CCA, which include chemical deterrence, overgrowth, and sloughing.

\section{Anti-settlement defense strategies of CCA}

Allelopathic compounds are employed by certain CCA species as natural antifoulants (Masaki et al.1981, Suzuki et al. 1998, Degnan and Johnson 1999). In the present study, extracts from each CCA species induced maximum settlement levels of $80 \%$ at low to medium extract concentrations (Fig. 4); however, higher concentrations (50 mg CCA/mL) inhibited settlement and metamorphosis. Larval elongation and reduced searching activity at the high extract concentrations indicated the presence of either nonspecific settlement inhibitors co-extracted by methanol, or dissolved allelopathic compounds. As coral larvae successfully settled on the live fragments of each of the CCA species tested, it is unlikely that these species contain or release allelopaths against coral settlement in environmentally effective concentrations.
Overgrowth has been described in several CCA species as a strategy to outcompete fouling organisms including newly settled corals (Babcock and Mundy 1996, Dunstan and Johnston 1998; see Results), and adult corals (Antonius 2001). However, our data indicate that overgrowth by CCA plays a relatively minor role in the survival of coral settlers, as the number of overgrowth events was small. For example, after 6 mo the density of coral spat on $T$. prototypum was $24 \%$ of the initial level, despite observed overgrowth of recruits by this CCA, and not significantly different from that on inorganic terracotta tiles $(20 \%)$.

Sloughing is a process by which some species of CCA shed off their upper epithallial layers thereby removing fouling organisms (Masaki et al. 1984, Keats et al. 1997). Sloughing of individual epithallial cells was the most effective antifouling strategy used by $N$. fosliei, $P$. onkodes, and $H$. reinboldii. Observations of incipient fouling in many species of living CCA, followed by the synchronous sloughing of whole epithallial layers (Sporolithon spp. and Neogoniolithon spp.) or a sloughing of individual epithallial cells in a spatially sequential fashion (Neogoniolithon mammillare) or diffuse fashion (Porolithon and Hydrolithon), indicate the importance of this process in CCA (Masaki et al. 1984, Johnson and Mann 1986). Similar antifouling strategies are widespread in marine and terrestrial plants; for example, seagrasses and laminarian kelps erode at the older apical regions to remove epibionts (Sand-Jensen and Borum 1991, Littler and Littler 1999). Scanning electron microscopy revealed that the epithalli of $T$. prototypum are usually intact, without peeling flakes. This anatomy and mode of growth appears to be a key to the high post-settlement survival on $T$. prototypum.

We are unable to compare the effectiveness of chemical defense against that of sloughing and overgrowth, as natural levels of allelopathic substances are unknown. The potentially allelopathic compounds co-extracted with the settlement inducers might primarily reduce the settlement of epibionts until the sloughing of epithallial cells of CCA surface occurs. Our combination of choice and no-choice settlement and survival experiments indicate that natural levels of allelopathy are insufficient to prevent coral settlement, that overgrowth is relatively ineffectual, and that sloughing is a highly effective strategy in preventing the survival of coral larvae on the five species of CCA tested.

\section{Larval ranking of potential settlement substrata}

We can now confirm that $T$. prototypum plays a critical role in influencing the fine-scale distribution patterns of coral recruits. Covering up to a few square centimeters, $T$. prototypum patches provide an excellent attachment surface for corals upon which competition and predation from other nonmotile reef organisms is low. However, T. prototypum comprises $<5 \%$ of the CCA flora on GBR reefs (R. Steneck, E. 
Turak, L. Harrington, and T. Done unpublished manuscript). So, rather than specializing in settlement exclusively onto a single uncommon CCA species, the larvae are able to actively recognize and select the next preferred substrata as suitable settlement sites, possibly keying into surface morphogens found in varying abundance or structure among CCA species (Fig. 5A). Hydrolithon borgesenii has been described as the key substratum for coral settlement in the Caribbean (Raimondi and Morse 2000). A similar species, H. reinboldii, is dominant throughout the tropical Indo-Pacific (Adey et al. 1982), and this species also contained a highly potent methanol-soluble morphogen and exhibited the second highest settlement and survival rates out of the remaining CCA species.

Habitat selection by planktonic larvae is critical to subsequent survival of sessile invertebrates, since location largely determines the environmental conditions experienced by later life stages (Keough and Downes 1982, Baird et al. 2003). Apart from recently disturbed and damaged reef surfaces, CCA offers a potentially favorable attachment site for disparate marine invertebrate taxa including coelenterates, which is stable and can facilitate survival and development to reproductive maturity. Among these settlers, the specificity of the interaction covers a spectrum, from species that manifest specificity for a particular species of CCA (Morse et al. 1996), to those requiring contact with any of a variety of CCA (Morse et al. 1988). We further demonstrated that coral larvae are able to actively recognize and rank different species of CCA as suitable settlement sites primarily based upon chemical signature and clearly reflecting the effectiveness of their antifouling strategies. This ranking process strongly supports the notion that settlement behavior (habitat selection) is adaptive and may be largely responsible for the finescale recruitment patterns observed in the field leading to a wider influence on coral reef structure.

\section{ACKNOWLEDGMENTS}

We gratefully acknowledge the help of Luke Smith, Greg Suosarri, Chico Birrell, Andrew Heyward, Harry Harrington, Tony Lamby, Juliane Metzner, and Claudia Vollhart for providing field assistance and participating during coral spawning. We are thankful for Lizard Island Research Station for providing facilities and support. We are also grateful to Robert Steneck, Andrew Baird, and Luke Smith for critical comments on the manuscript. L. Harrington acknowledges the support of an International Postgraduate Research Scholarship Award from the James Cook University. The research was funded by the Cooperative Research Centre for the Great Barrier Reef World Heritage Area (CRC Reef) and the Australian Institute of Marine Science (AIMS).

\section{Literature Cited}

Adey, W. H., R. A. Townsend, and W. T. Boykins. 1982. The crustose coralline algae (Rhodophyta: Corallinaceae) of the Hawaiian Islands. Smithsonian Contributions to the Marine Sciences 15:1-74.

Airoldi, L. 2000. Effects of disturbance, life histories, and overgrowth on coexistence of algal crusts and turfs. Ecology 81:798-814.
Antonius, A. 2001. Pneophyllumm conicum, a coralline red algae causing coral reef-death in Mauritius. Coral Reefs 19:418.

Babcock, R., and C. Mundy. 1996. Coral recruitment: consequences of settlement choice for early growth and survivorship in two scleractinians. Journal of Experimental Marine Biology and Ecology 206:179-201.

Baird, A. H., R. C. Babcock, and C. P. Mundy. 2003. Habitat selection by larvae influences the depth distribution of six common coral species. Marine Ecology Progress Series 252:289-293.

Baird, A. H., and T. Hughes. 2000. Competitive dominance by tabular corals: an experimental analysis of recruitment and survival of understory assemblages. Journal of Experimental Marine Biology and Ecology 251:117-132.

Baird, A. H., and A. N. C. Morse. 2004. Induction of metamorphosis in larvae of the brooding corals Acropora palifera and Stylophora pistillata. Marine and Freshwater Research 55:469-472.

Davison, A. C., and D. V. Hinkley. 1997. Bootstrap methods and their application. Cambridge University Press, Cambridge, UK.

Degnan, B. M., and C. R. Johnson. 1999. Inhibition of settlement and metamorphosis of the ascidian Herdmania curvata by non-geniculate coralline algae. Biological Bulletin 197:332-340.

Dunstan, P. K., and C. R. Johnson. 1998. Spatio-temporal variation in coral recruitment at different scales on Heron Reef, southern Great Barrier Reef. Coral Reefs 17:71-81.

Ehrenberg, C. G. 1834. Beiträge zur physiologischen Kentnissder Corallenthiere im allgemeinen, und besonders des rothen Meeres, nebst einem Versuche Zur Physiologischen Systematik derselben. Abhandlungen dur Königlichen Akademie der Wissenschaften zu Berlin 1:225-380.

Gordon, G. D., T. Masaki, and H. Akioka. 1976. Floristic and distributional account of the common crustose coralline algae on Guam. Micronesica 12:247-277.

Grosberg, R. K. 1981. Competitive ability influences habitat choice in marine invertebrates. Nature 290:700-702.

Hastie, T. J., and R. J. Tibshirani. 1990. Generalized additive models. Chapman and Hall, London, UK.

Heyward, A. J., and A. P. Negri. 1999. Natural inducers for coral larval metamorphosis. Coral Reefs 18:273-279.

Johnson, C. R., T. E. Lewis, D. S. Nichols, and B. M. Degnan. 1997. Bacterial induction of settlement and metamorphosis in marine invertebrates. Proceeding of the eighth International Coral Reef Symposium 2:1219-1224.

Johnson, C. R., and K. H. Mann. 1986. The crustose coralline algae Phymatolithon Foslie, inhibits overgrowth of seaweeds without relying on herbivores. Journal of Experimental Marine Biology and Ecology 96:127-146.

Keats, D. W., M. A. Knight, and C. M. Pueschel. 1997. Antifouling effects of epithallial shedding in three crustose coralline algae (Rhodophyta, Corallinales) on a coral reef. Journal of Experimental Marine Biology and Ecology 213: 281-293.

Keough, M., and J. Downes. 1982. Recruitment of marine invertebrates: the role of active larval choices and early mortality. Oecologia 54:348-352.

Littler, M., and D. S. Littler. 1999. Blade abandonment/proliferation: a novel mechanism for rapid epiphyte control in marine macrophytes. Ecology 80:1736-1746.

Maida, M., J. C. Coll, and P. W. Sammarco. 1994. Shedding new light on Scleractinian coral recruitment. Journal of Experimental Marine Biology and Ecology 180:189-202.

Maida, M., P. W. Sammarco, and J. C. Coll. 1995. Effects of soft corals on Scleractinian coral recruitment. I: directional allelopathy and inhibition of settlement. Marine Ecology Progress Series 121:191-202. 
Marsh, J. 1970. Primary productivity of reef-building calcareous red algae. Ecology 51:255-263.

Masaki, D., D. Fujita, and H. Akioka. 1981. Observation on the spore germination of Laminaria japonica on Lithophyllum yessoense (Rhodophyta, Corallinaceae) in culture. Bulletin of the Faculty of Fisheries, Hokkaido University 32:349-356.

Masaki, D., D. Fujita, and N. T. Hagen. 1984. The surface ultrastructure and epithallium shedding of crustose coralline algae in an 'Isoyake' area of southwestern Hokkaido, Japan. Hydrobiologia 116/117:218-223.

McCullagh, P., and J. A. Nelder. 1989. Generalized linear models. Second edition. Chapman and Hall, London, UK.

Morse, A. N. C., K. Iwao, M. Baba, K. Shimoike, T. Hayashibara, and M. Omori. 1996. An ancient chemosensory mechanism brings new life to coral reefs. Biological Bulletin 191:149-154.

Morse, A., and D. E. Morse. 1984. Recruitment and metamorphosis of Haliotis larvae induced by molecules uniquely available at the surfaces of crustose red algae. Journal of Experimental Marine Biology and Ecology 75:191-215.

Morse, D. E., N. Hooker, A. N. C. Morse, and R. A. Jensen. 1988. Control of larval metamorphosis and recruitment in sympatric agariciid corals. Journal of Experimental Marine Biology and Ecology 116:193-217.

Morse, D. E., and A. N. C. Morse. 1991. Enzymatic characterization of the morphogen recognized by Agaricia humilis (scleractinian coral) larvae. Biological Bulletin 181: 104-122.

Mundy, C. N., and R. C. Babcock. 1998. Role of light intensity and spectral quality in coral settlement: implications for depth-dependent settlement. Journal of Experimental Marine Biology and Ecology 223:235-255.

Palma, A. T., R. S. Steneck, and C. J. Wilson. 1999. Settlement driven, multiscale demographic patterns of large ben- thic decapods in the Gulf of Maine. Journal of Experimental Marine Biology and Ecology 241:107-136.

Raimondi, P. T. 1990. Patterns, mechanisms, consequences of variability in settlement and recruitment of an intertidal barnacle. Ecological Monographs 60:85:283-309.

Raimondi, P. T., and A. N. C. Morse. 2000. The consequences of complex larval behavior in a coral. Ecology 81:31933211.

Ruiz-Zarate, M., J. E. D. Fragosa, and J. P. Carricart-Ganivet. 2000. Relationships between Maicina areloata (Cnidaria: Scleractinia), Thalassia testudinum (Anthophyta) and Neogoniolithon sp. (Rhodophyta). Marine Ecology Progress Series 206: $135-146$.

Sand-Jesen, K., and J. Borum. 1991. Interactions among phytoplankton, periphyton, and macrophytes in temperate freshwaters and estuaries. Aquatic Botany 41:137-175.

Sebens, K. 1986. Spatial relationships among encrusting marine organisms in the New England subtidal zone. Ecological Monographs 56:73-96.

Suzuki, Y., T. Takabayashi, T. Kawaguchi, and K. Matsunaga. 1998. Isolation of an allelopathic substance from the crustose coralline algae, Lithophyllum spp., and its effects on the brown alga, Laminaria religiosa Miyabe (Phaeophyta). Journal of Experimental Marine Biology and Ecology 225: 69-77.

Webster, N. S., L. D. Smith, A. J. Heyward, J. M. E. Watts, R. I. Webb, L. L. Blackall, and A. P. Negri. 2004. Metamorphosis of a scleractinian coral in response to microbial biofilms. Applied and Environmental Microbiology 70: 1213-1221.

Young, C. M., and F. S. Chai. 1985. Abundance and distribution of pelagic larvae as influenced by predation, behavior and hydrodynamic factors. Pages 385-463 in A. C. Giese and J. S. Pearse, editors. Reproduction of marine invertebrates. Academic Press, New York, New York, USA.

\section{APPENDIX}

A photograph showing examples of anti-settlement defenses in crustose coralline algae is available in ESA's Electronic Data Archive: Ecological Archives E085-120-A1. 